\title{
Using an ensemble of statistical metrics to quantify large sets of plant transcription factor binding sites
}

\author{
Parsa Hosseini ${ }^{1,2,3^{*}}$, Ivan Ovcharenko ${ }^{2}$ and Benjamin $\mathrm{F} \mathrm{Matthews}^{3}$
}

\begin{abstract}
Background: From initial seed germination through reproduction, plants continuously reprogram their transcriptional repertoire to facilitate growth and development. This dynamic is mediated by a diverse but inextricably-linked catalog of regulatory proteins called transcription factors (TFs). Statistically quantifying TF binding site (TFBS) abundance in promoters of differentially expressed genes can be used to identify binding site patterns in promoters that are closely related to stress-response. Output from today's transcriptomic assays necessitates statistically-oriented software to handle large promoter-sequence sets in a computationally tractable fashion.

Results: We present Marina, an open-source software for identifying over-represented TFBSs from amongst large sets of promoter sequences, using an ensemble of 7 statistical metrics and binding-site profiles. Through software comparison, we show that Marina can identify considerably more over-represented plant TFBSs compared to a popular software alternative.

Conclusions: Marina was used to identify over-represented TFBSs in a two time-point RNA-Seq study exploring the transcriptomic interplay between soybean (Glycine max) and soybean rust (Phakopsora pachyrhizi). Marina identified numerous abundant TFBSs recognized by transcription factors that are associated with defense-response such as WRKY, HY5 and MYB2. Comparing results from Marina to that of a popular software alternative suggests that regardless of the number of promoter-sequences, Marina is able to identify significantly more over-represented TFBSs.
\end{abstract}

\section{Background}

\section{Definitions and presumptions}

We define a list of transcription factor binding sites (TFBSs), $t_{1}, t_{2}, \ldots, t_{N}$, where $t_{i}$ is either a DNA motif, $m_{i}$ or position weight matrix (PWM), $w_{i}$. The former is a variable-length character-string from the four-nucleotide DNA alphabet, while the latter is a two-dimensional matrix of preset weights.

A group, $G_{a}$, is a FASTA file populated with userprovided promoter sequences. Let $G_{a}, G_{a+1}, \ldots, G_{N}$ represent a list of $N$ groups such that $N \geq 2$. We define a contingency matrix, $c_{i}$ as a $2 \times 2$ matrix, used to model

\footnotetext{
*Correspondence: parsa.hosseini@nih.gov

${ }^{1}$ Department of Bioinformatics and Computational Biology, George Mason

University, Manassas, Virginia, USA

${ }^{2}$ Computational Biology Branch, National Center for Biotechnology Information, National Institutes of Health, Bethesda, Maryland, USA Full list of author information is available at the end of the article
}

$t_{i}$ over-representation across $G_{a}$ and $G_{a+1}$. A set of statistical metrics, $S$, quantify degree of $t_{i}$ over-representation given $c_{i}$.

\section{Transcription factors and binding site representation}

Plants are constantly surrounded by stimulus, be-they deletorious pathogens or positive stimuli such as light and nutrients. In order for the plant to respond to these signals, plants must utilize regulatory proteins known as transcription factors (TFs) to facilitate transcriptional reprogramming in a dynamic, tissue-dependent manner. These proteins bind to enhancer or promoter cis-elements and facilitate the recruitment of RNA polymerase II. This combinatorial binding of TFs facilitates downstream execution of adaptative signals in the face of drought, herbivory, and high salinity. By quantifying binding-sites for these regulatory proteins, inherent transcriptional dynamics and magnitude of over-representation can be inferred. 
TFs are classified into families by inherent DNA-binding signatures otherwise known as protein domains. In Arabidopsis thaliana, for instance, there are 64 known TF families [1], and it is not uncommon for different TF family members to exhibit relatively similar functionality. This redundancy is especially true when it comes to stress-response [2-4].

DNA motifs and PWMs are two models frequently used to define a TFBS. The former is a short cis-element region presumed to be a TFBS, while the latter models nucleotide propensities of a TFBS in the form of a matrix $[5,6]$. PWMs have been used across a broad spectrum of plant investigations such as identification of conserved exonic splice-site enhancers in Arabidopsis thaliana [7], prediction of potential seed-storage regulatory elements in mustards, grasses and legumes [8], and identification of novel regulatory elements in Arabidopsis thaliana [9]. With assays such as ChIP-ChIP and ChIP-Seq, novel regulatory elements can be identified and modeled as a PWM [10].

\section{Implementation}

Marina is an operating-system independent GUI software tool built using the Java programming language.

This manuscript builds on the works of Chekmenev et. al [11], Loots et. al [12], and Kel et. al. [13], by implementing multiple statistical metrics to identify the maximum number of biologically-sound TFBSs, while accounting for cases when large promoter sets are provided.

To begin analysis with Marina, at least two FASTA files populated with user-provided promoter sequences are required. Each FASTA file is known as a group. A group, for instance, could represent promoter sequences of interest for a particular condition or time point.

The Marina workflow (Figure 1) is partitioned into three distinct phases. The first phase performs abundanceestimation given a catalog of known TFBS models (Figure 1a). Initial abundance derivation is performed via mapping of the TFBS onto user-provided promoter sequences. Following TFBS mapping, low-quality TFBSs are removed (Figure 1b). Finally, a collection of statistical metrics quantify and rank TFBS over-representation (Figure 1c).

\section{Phase 1: Binding site mapping}

In order to effectively quantify TFBS abundance using this tool, TFBS models must be provided. These models are in the form of either DNA motifs or PWMs. Cumulatively, 1,240 TFBS models were mined and utilized throughout this study. Of these models, 1,160 were DNA motifs with the remaining 80 being PWMs; motif-to-PWM ratio of $13: 1$.

Plant DNA motif and PWM models originated from AthaMap [14], AGRIS [15], PlantCARE [16], TRANSFAC
[17], and JASPAR [18]. DNA motifs and PWMs were stored in either a tab-delimited or FASTA file format, respectively. Due to licensing restrictions, Marina does not come pre-packaged with a catalog of TFBS models, however several PWMs are provided, built from known PDB structures using the 3DTF web-server [19]. Be it PWMs or DNA motifs, a user-friendly schema is provided for importing custom TFBS profiles.

\section{DNA motif and PWM mapping}

To efficiently derive over-representation using DNA motifs, Marina scans promoter sequences for any occurrence of this motif using the Boyer-Moore-Horspool algorithm [20]. Due to the short length of many DNA motifs, elements such as ARF1 (TGTCTC) [21] may ubiquitously map throughout a promoter sequence with many mappings having little biological significance. Though this tool provides the option to filter short-length models be it PWMs or DNA motifs, resultant abundance estimations may seldom be biologically significant simply due to the likelihood of spurious mappings.

Marina maps each PWM onto promoter sequences using a concurrent implementation of the P-MATCH algorithm [11]. P-MATCH calculates a likelihood that a particular candidate promoter region contains a TFBS. By default, Marina uses a probability-cutoff of 0.80; any subsequence with a score greater than this cutoff is rendered a potential TFBS.

Alongside DNA motif and PWM extrapolations is a third pseudo-extrapolation known as combined mode. This mode simply performs the two former extrapolations back-to-back, merging their results into a singular data-structure. Combined mode capitalizes on the abundance of DNA motifs and probabilistic power of PWMs.

\section{Phase 2: Modeling TFBS over-representation}

TFBS abundances across all promoter sequences are modeled using a group-specific acyclic graph. Each graph is organized such that group name is the rootnode and each TFBS is a child leaf node. Every TFBS node references a list of promoter sequences containing this TFBS.

Per graph child node, two measures are used to model initial TFBS abundance: raw counts and support [22]. The former is simply defined as the number of promoter sequences which contain this particular TFBS. Raw counts are a useful, comparable metric if all groups have approximately the same number of promoter sequences. Unfortunately some groups may be larger than others, resulting in skewed and uncontrastable counts. To circumvent this possibility, the latter probabilistic measure, support, comes in helpful. Support, $P\left(t_{i}, G_{a}\right)$, is a data-mining metric for 


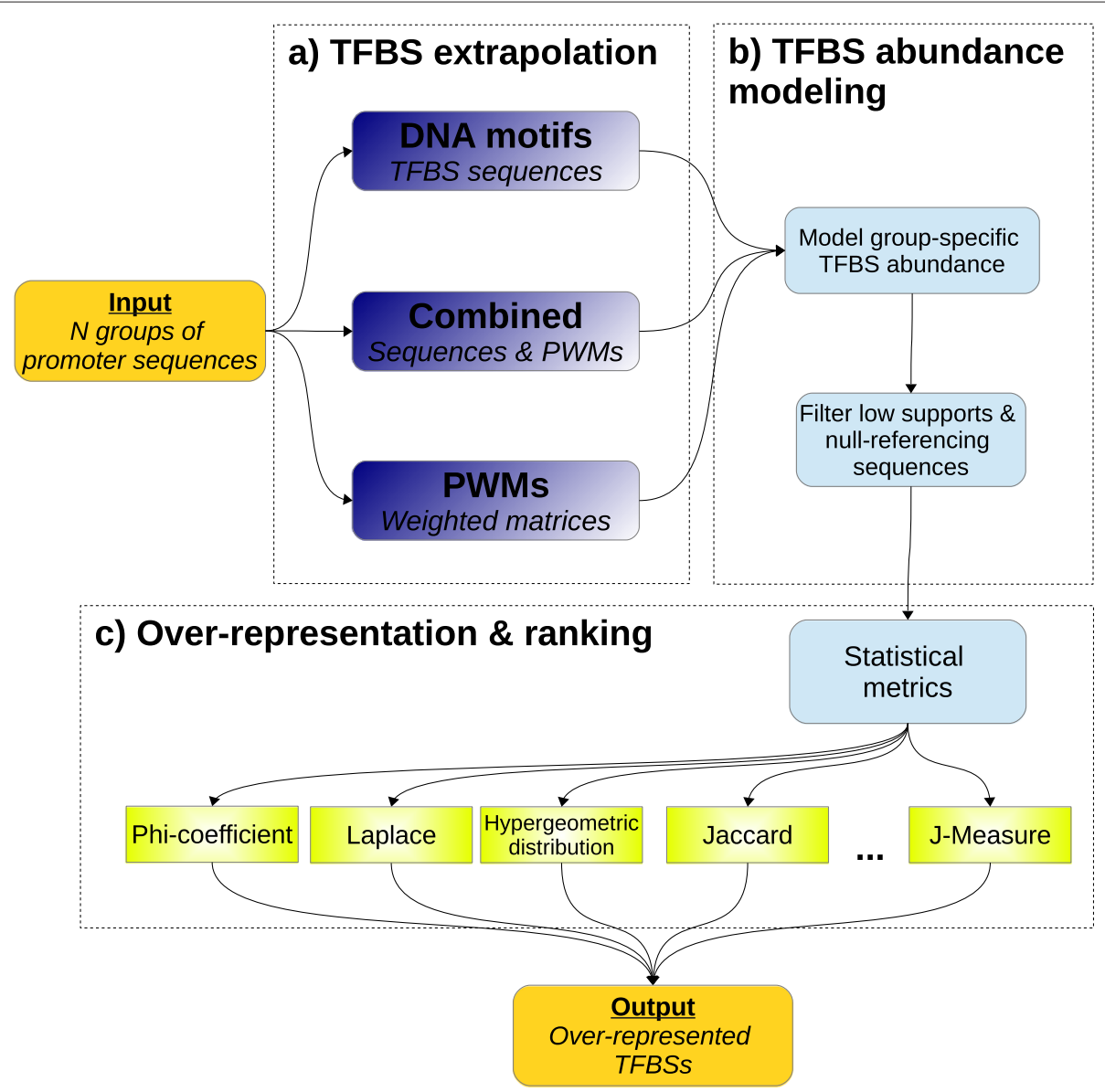

Figure 1 Marina workflow. a) A group is an umbrella-term to represent a set of promoter sequences. In order to run Marina, at least two groups must be provided. In doing so, TFBSs within each group can be contrasted and statistically quantified using TFBSs modeled as either DNA motifs or PWMs. Marina can also run if both these data-structures are provided, hence the name combined. b) Each group is modeled as a uni-directional graph, providing a means of trimming low-abundant promoter-sequences and TFBSs. c) A diverse collection of statistical metrics are used to model and quantify TFBS abundance. Magnitude of TFBS abundance is ranked and the hypergeometric distribution p-value computes significance of TFBS over-representation.

representing abundance of a TFBS within a particular group [22]. A collection of statistical metrics continue where support leaves off, providing a means of deducing TFBS abundance.

Both raw-counts and support serve as viable metrics to initially model TFBS abundance, however there may be cases were a rift between the two measures can appear. For example, suppose a single TFBS mapped only once to a group. Due to such minimal mapping, raw-count will be small but support would be large. Both low-support and low-count thresholds exist so as to filter corresponding graph nodes. Such graph trimming ensures that high-support and/or high-count TFBS nodes remain as they are more likely of having correlations to a particular group [23]. A caveat with threshold cutoffs is that low-abundance TFBSs will get discarded.
Phase 3: Deriving over-represented TFBSs using numerous statistical metrics

Given remaining TFBSs nodes, Marina aims to deduce magnitude of over-representation per TFBS, $t_{i}$ by contrasting its abundance across groups $G_{a}$ and $G_{a+1}$. To facilitate this objective, a collection of 7 knowledge discovery metrics, $S$, are implemented (Table 1 ). Though a single metric can theoretically suffice, employing the entire set provides a means to appreciate unique features per measure and avoid individual bias. This table is by no means exhaustive as there are well over 20 frequently used metrics $[24,25]$. The metrics in this table were selected so that there exists a sound mixture of both well-studied association and correlation measures.

In order to utilize such measures, TFBS abundances must be modeled in a suitable data-structure. A contingency matrix, $c_{i}$, is an ideal data-structure 
Table 1 Statistical metrics

\begin{tabular}{|c|c|c|c|}
\hline Metric & Equation & Output range & Reference \\
\hline Confidence (CF) & $\max \left(P\left(G_{a} \mid t_{i}\right), P\left(t_{j} \mid G_{a}\right)\right)$ & $0 \ldots 1$ & [26] \\
\hline Cosine $(\mathrm{CO})$ & $\frac{P\left(t_{i}, G_{a}\right)}{\sqrt{P\left(t_{i}\right) P\left(G_{a}\right)}}$ & $0 \ldots \sqrt{P\left(t_{i}, G_{a}\right)} \ldots 1$ & [27] \\
\hline Jaccard (JAC) & $\frac{P\left(t_{i}, G_{a}\right)}{P\left(t_{i}\right)+P\left(G_{a}\right)-P\left(t_{i}, G_{a}\right)}$ & $0 \ldots 1$ & {$[28]$} \\
\hline Kappa coefficient (K) & $\frac{\left.P\left(t_{i}, G_{a}\right)+P\left(\overline{\left(t_{i}, G_{a}\right.}\right)-P\left(t_{i}\right) P\left(G_{a}\right)-P\left(\overline{t_{i}}\right) P\left(\overline{G_{a}}\right)\right)}{1-P\left(t_{i}\right) P\left(G_{a}\right)-P\left(\bar{t}_{i}\right) P\left(\overline{G_{a}}\right)}$ & $-1 \ldots 1$ & [29] \\
\hline Laplace Correction (LP) & $\max \left(\frac{N P\left(t_{i}, G_{a}\right)+1}{N P\left(t_{i}\right)+2}, \frac{N P\left(t_{i}, G_{a}\right)+1}{N P\left(G_{a}\right)+2}\right)$ & $0 \ldots 1$ & {$[30]$} \\
\hline Lift (LI) & $\frac{P\left(t_{i}, G_{a}\right)}{P\left(t_{i}\right) P\left(G_{a}\right)}$ & $0 \ldots \infty$ & [31] \\
\hline Phi coefficient (PHI) & $\frac{P\left(t_{i}, G_{a}\right)-P\left(t_{i}\right) P\left(G_{a}\right)}{\sqrt{P\left(t_{i}\right) P\left(G_{a}\right)\left(1-P\left(t_{i}\right)\right)\left(1-P\left(G_{a}\right)\right)}}$ & $-1 \ldots 1$ & {$[32]$} \\
\hline
\end{tabular}

Given a group, $G_{a}$, and a TFBS, $t_{i}$, magnitude of TFBS over-representation can be determined using a set of statistical metrics.

candidate as it models TFBS distributions throughout multiple, independent groups (Table 2). Each metric within $S$ processes frequencies within a contingency matrix, $c_{i}$, so as to quantitatively deduce overrepresentation of TFBS, $t_{i}$. Certainly not all metrics deduce magnitude of TFBS over-representation the same, resulting in difficulties as to which TFBSs are unanimously most over-represented by all metrics. A solution to bringing uniform over-representation agreement across all metrics is to standardize contingency matrix counts using Iterative Proportional Fitting (IPF) [33].

\section{Iterative Proportional Fitting (IPF)}

IPF is an algorithm for standardizing counts in a two-dimensional contingency matrix such that matrix row and column marginals are equal to one another (Table 3). Through such adjustment, inherent associations and correlations can be discovered [34]. By performing IPF-standardization, output for all 7 metrics become normalized so as to agree which TFBSs are the most over-represented.

Equations 1 and 2 present an implementation of the IPF algorithm originally outlined by Tan et al. [35]. The former equation adjusts counts, $a$, such that they are equal on the diagonal axis. The latter equation then subtracts the remainder of the counts from that of the entire matrix sum, $N$.

$$
\begin{aligned}
& c_{i 1,0}=c_{i 0,0}=a=\frac{N \sqrt{c_{i 1,1} c_{i 0,0}}}{2\left(\sqrt{c_{i 1,1} c_{i 0,0}}+\sqrt{c_{i 1,0} c_{i 0,1}}\right)} \\
& c_{i 0,1}=c_{i 1,0}=\frac{N}{2}-a
\end{aligned}
$$

Table 2 Contingency matrices model TFBS over-representation

\begin{tabular}{cccc}
\hline & $\boldsymbol{G}_{\boldsymbol{a}}$ & $\overline{\boldsymbol{G}_{\boldsymbol{a}}}$ & \\
\hline$t_{i}$ & $c_{i}(0,0)$ & $c_{i}(1,0)$ & $n\left(t_{i}\right)$ \\
$\overline{t_{i}}$ & $c_{i}(0,1)$ & $c_{i}(1,1)$ & $n\left(\overline{t_{i}}\right)$ \\
\hline & $n\left(G_{a}\right)$ & $n\left(\overline{G_{a}}\right)$ & $N$ \\
\hline
\end{tabular}

TFBS abundance within specific groups can be modeled as a two-dimensional contingency matrix, $c_{j}$.

\section{Results and discussion}

Case study: over-represented Glycine max TFBSs during a Phakopsora pachyrhizi time-course infection

To evaluate the functionality of this software tool, we utilized a two time-course RNA-Seq study that investigates soybean (Glycine max) transcriptional dynamics upon pathogenesis with soybean rust (SR; Phakopsora pachyrhizi). As outlined in our previous study, susceptible Williams 82 soybean leaves were inoculated with SR and assayed using RNA-Seq 10 days after infection (dai) [36]. An accompanying uninoculated control was also assayed to serve as a baseline condition. In both the control and 10 dai samples, a total of 5,940,995 70bp reads and $5,574,89240 \mathrm{bp}$ reads were respectively sequenced using the Illumina platform (GenomeAnalyzer IIx). Sequenced reads were deposited in NCBI SRA under accessions SRX100854, SRX129967 and SRX100853, SRX129959, respectively.

Per run, quality assessment and control (QA/QC) entailed removal of low quality reads and trimming of low-quality 3' ends should its quality score be less than 22. Reads were also discarded if they mapped at least once to either the human genome (Hg19) or the JCVI Microbial Resource [37]. Upon QA/QC completion, a total of 5,015,459 control reads and 5,420,745 10 dai reads passed filtering; quality-scores of 27 and 30, respectively. For each time point, reads were mapped with at-most 3 nucleotide mismatches onto the soybean transcriptome build (Glyma 1.0) using BWA [38]. Custom Python scripts inferred differential expression by deriving RPKM [39] and $\log _{2}\left(\frac{R P K M_{10 d a i}}{R P K M_{0 d a i}}\right)$ per transcript.

Table 3 IPF-standardization yields equal marginals in a contingency matrix

\begin{tabular}{cccc}
\hline & $\boldsymbol{G}_{\boldsymbol{a}}$ & $\overline{\boldsymbol{G}_{\boldsymbol{a}}}$ & \\
\hline$t_{i}$ & $x$ & $N / 2-x$ & $N / 2$ \\
$\overline{t_{i}}$ & $N / 2-x$ & $x$ & $N / 2$ \\
\hline & $N / 2$ & $N / 2$ & $N$ \\
\hline
\end{tabular}

Identical counts within diagonal cells leads to marginal rows and columns being equal to one another. Table adapted from [35]. 
Table 4 Various metrics infer differing magnitudes of TFBS over-representation

\begin{tabular}{|c|c|c|c|c|c|c|c|c|c|c|}
\hline \multicolumn{9}{|c|}{ Metrics } & \multicolumn{2}{|c|}{ TFBS raw-abundance } \\
\hline TF & LP & $\mathrm{CO}$ & JAC & LI & $\mathrm{CF}$ & K & PHI & p-value & Suppressed & Induced \\
\hline ABF1 & 20 & 39 & 39 & 20 & 20 & 3 & 2 & $8.211 e-274$ & 130 & 169 \\
\hline ABFS & 9 & 9 & 10 & 9 & 9 & 16 & 12 & $2.385 e-31$ & 10 & 20 \\
\hline ABI3/FUS3 & 67 & 19 & 17 & 67 & 67 & 41 & 58 & $3.036 \mathrm{e}-47$ & 14 & 7 \\
\hline$A B \mid 4(2)$ & 64 & 34 & 33 & 64 & 64 & 64 & 67 & $4.465 e-172$ & 66 & 43 \\
\hline$A G$ & 14 & 20 & 21 & 14 & 14 & 13 & 18 & $4.611 \mathrm{e}-82$ & 30 & 42 \\
\hline AGP1 & 48 & 57 & 56 & 48 & 48 & 58 & 49 & $2.412 \mathrm{e}-720$ & 427 & 398 \\
\hline ALFIN1 & 34 & 58 & 57 & 34 & 34 & 34 & 34 & $1.580 \mathrm{e}-731$ & 440 & 426 \\
\hline ARF1 & 65 & 29 & 24 & 65 & 65 & 57 & 62 & $1.243 e-113$ & 40 & 25 \\
\hline ARR10 & 39 & 65 & 65 & 39 & 39 & 43 & 39 & $1.836 \mathrm{e}-895$ & 579 & 552 \\
\hline ARR2 & 69 & 27 & 22 & 69 & 69 & 60 & 69 & $4.028 \mathrm{e}-99$ & 33 & 15 \\
\hline ATHB-5 & 43 & 68 & 68 & 43 & 43 & 49 & 43 & $1.542 \mathrm{e}-901$ & 584 & 555 \\
\hline ATHB1 & 40 & 67 & 67 & 40 & 40 & 45 & 40 & $3.162 e-901$ & 584 & 556 \\
\hline ATHB5-1 & 63 & 21 & 20 & 63 & 63 & 44 & 55 & $3.202 \mathrm{e}-78$ & 26 & 18 \\
\hline ATHB5-2 & 37 & 60 & 60 & 37 & 37 & 37 & 37 & $9.771 e-769$ & 470 & 452 \\
\hline ATHB6 & 27 & 23 & 25 & 27 & 27 & 29 & 32 & $3.067 e-109$ & 41 & 46 \\
\hline ATHB9 & 53 & 38 & 36 & 53 & 53 & 55 & 52 & $2.105 e-225$ & 95 & 81 \\
\hline AtLEC2 & 55 & 51 & 51 & 55 & 55 & 68 & 61 & $1.066 \mathrm{e}-611$ & 336 & 284 \\
\hline ATML1/PDF2 & 71 & 18 & 11 & 71 & 71 & 54 & 71 & $8.730 e-38$ & 10 & 1 \\
\hline AtMYB2 & 29 & 33 & 34 & 29 & 29 & 23 & 31 & $1.606 \mathrm{e}-170$ & 70 & 76 \\
\hline AtMYB77 & 60 & 32 & 31 & 60 & 60 & 56 & 57 & $2.955 e-141$ & 53 & 40 \\
\hline AtMYC2 & 2 & 2 & 2 & 2 & 2 & 30 & 8 & 0.0002735 & 1 & 7 \\
\hline AtSPL3 & 30 & 45 & 46 & 30 & 30 & 8 & 26 & 7.997e-426 & 220 & 236 \\
\hline BLR/RPL/PNY & 35 & 61 & 61 & 35 & 35 & 35 & 35 & $1.444 \mathrm{e}-777$ & 478 & 462 \\
\hline bZIP910(2) & 10 & 12 & 16 & 10 & 10 & 14 & 11 & $6.060 \mathrm{e}-42$ & 14 & 26 \\
\hline bZIP911 & 12 & 11 & 13 & 12 & 12 & 19 & 14 & $4.350 e-37$ & 12 & 21 \\
\hline bZIP911(1) & 11 & 10 & 12 & 11 & 11 & 20 & 13 & $2.529 \mathrm{e}-34$ & 11 & 20 \\
\hline bZIP911(2) & 18 & 13 & 14 & 16 & 16 & 32 & 29 & $3.730 e-38$ & 12 & 16 \\
\hline $\mathrm{CBF}$ & 43 & 68 & 68 & 43 & 43 & 49 & 43 & $1.542 \mathrm{e}-901$ & 584 & 555 \\
\hline CDC5 & 4 & 4 & 4 & 4 & 4 & 18 & 3 & $1.343 e-10$ & 3 & 13 \\
\hline DOF2 & 42 & 71 & 71 & 42 & 42 & 48 & 42 & $1.259 e-902$ & 585 & 556 \\
\hline DPBF1/2 & 51 & 55 & 55 & 51 & 51 & 66 & 54 & $1.857 \mathrm{e}-712$ & 418 & 379 \\
\hline $\mathrm{E} 2 \mathrm{Fa}$ & 70 & 13 & 9 & 70 & 70 & 38 & 64 & $8.059 e-24$ & 6 & 1 \\
\hline $\mathrm{E} 2 \mathrm{Fc} / \mathrm{d}$ & 1 & 1 & 1 & 1 & 1 & 26 & 5 & 0.0003077 & 1 & 8 \\
\hline EmBP-1 & 25 & 43 & 43 & 25 & 25 & 5 & 17 & $3.316 e-397$ & 203 & 228 \\
\hline GAMYB & 47 & 59 & 59 & 47 & 47 & 53 & 47 & $2.040 e-743$ & 447 & 422 \\
\hline Gamyb & 58 & 28 & 26 & 58 & 58 & 40 & 50 & $6.185 e-120$ & 44 & 36 \\
\hline GATA-1 & 17 & 24 & 28 & 18 & 18 & 12 & 16 & $5.923 e-120$ & 47 & 62 \\
\hline GATA-1/2/3/4 & 16 & 15 & 18 & 17 & 17 & 28 & 27 & $9.291 \mathrm{e}-54$ & 18 & 24 \\
\hline GT-3b & 13 & 25 & 29 & 13 & 13 & 7 & 7 & $1.244 \mathrm{e}-128$ & 52 & 76 \\
\hline HAHB4 & 46 & 64 & 64 & 46 & 46 & 52 & 46 & $1.038 \mathrm{e}-891$ & 575 & 546 \\
\hline HAT5 & 43 & 68 & 68 & 43 & 43 & 49 & 43 & $1.542 \mathrm{e}-901$ & 584 & 555 \\
\hline HSE & 19 & 26 & 30 & 19 & 19 & 11 & 15 & $1.641 \mathrm{e}-130$ & 52 & 68 \\
\hline $\mathrm{HVH} 21$ & 41 & 66 & 66 & 41 & 41 & 46 & 41 & 3.881 e-900 & 583 & 555 \\
\hline
\end{tabular}


Table 4 Various metrics infer differing magnitudes of TFBS over-representation (Continued)

\begin{tabular}{|c|c|c|c|c|c|c|c|c|c|c|}
\hline HY5 & 6 & 8 & 8 & 6 & 6 & 21 & 10 & $6.247 e-20$ & 6 & 15 \\
\hline ID1 & 28 & 31 & 32 & 28 & 28 & 27 & 33 & $4.015 e-146$ & 58 & 63 \\
\hline MYB.PH3(1) & 56 & 41 & 41 & 56 & 56 & 61 & 56 & $4.159 e-333$ & 154 & 130 \\
\hline MYB.PH3(2) & 52 & 49 & 49 & 52 & 52 & 62 & 53 & $6.937 e-564$ & 306 & 276 \\
\hline MYB98 & 62 & 36 & 35 & 62 & 62 & 65 & 65 & $2.648 \mathrm{e}-210$ & 85 & 60 \\
\hline $\mathrm{O} 2$ & 33 & 56 & 58 & 33 & 33 & 6 & 28 & 2.967e-731 & 446 & 457 \\
\hline OsbHLH66 & 26 & 40 & 40 & 26 & 26 & 9 & 20 & $1.723 e-308$ & 147 & 165 \\
\hline OsCBT & 3 & 3 & 3 & 3 & 3 & 24 & 6 & $1.543 e-7$ & 2 & 10 \\
\hline$P$ & 57 & 52 & 52 & 57 & 57 & 71 & 66 & $2.571 e-629$ & 347 & 286 \\
\hline PCF2 & 61 & 47 & 44 & 61 & 61 & 70 & 70 & $3.566 \mathrm{e}-441$ & 215 & 160 \\
\hline PCF5 & 59 & 48 & 48 & 59 & 59 & 69 & 68 & $2.612 \mathrm{e}-498$ & 254 & 201 \\
\hline PEND & 31 & 35 & 37 & 31 & 31 & 15 & 30 & $3.825 e-230$ & 101 & 108 \\
\hline PIF3(2) & 21 & 22 & 23 & 21 & 21 & 17 & 25 & $4.178 \mathrm{e}-99$ & 37 & 46 \\
\hline RAP2.2 & 66 & 30 & 27 & 66 & 66 & 59 & 63 & $1.614 \mathrm{e}-125$ & 45 & 28 \\
\hline RAV1(1) & 49 & 54 & 53 & 49 & 49 & 63 & 51 & $1.957 e-688$ & 400 & 366 \\
\hline RAV1(2) & 38 & 62 & 62 & 38 & 38 & 39 & 38 & $1.073 e-854$ & 543 & 519 \\
\hline STF1 & 24 & 37 & 38 & 24 & 24 & 10 & 22 & $1.243 e-242$ & 109 & 124 \\
\hline TAC1 & 68 & 17 & 15 & 68 & 68 & 42 & 59 & $1.479 e-44$ & 13 & 6 \\
\hline TaMYB80 & 54 & 50 & 50 & 54 & 54 & 67 & 60 & $2.700 e-594$ & 324 & 276 \\
\hline TBP & 36 & 63 & 63 & 36 & 36 & 36 & 36 & $1.545 e-881$ & 568 & 547 \\
\hline TEIL & 50 & 42 & 42 & 50 & 50 & 47 & 48 & $8.458 \mathrm{e}-340$ & 160 & 146 \\
\hline TGA1 & 23 & 46 & 47 & 23 & 23 & 2 & 9 & $3.416 e-468$ & 253 & 293 \\
\hline TGA1a & 32 & 53 & 54 & 32 & 32 & 4 & 23 & $3.325 e-688$ & 413 & 433 \\
\hline WRKY11 & 7 & 7 & 7 & 8 & 8 & 31 & 19 & $2.346 \mathrm{e}-14$ & 4 & 9 \\
\hline WRKY18/40/62 & 7 & 6 & 6 & 7 & 7 & 33 & 21 & $2.879 \mathrm{e}-11$ & 3 & 7 \\
\hline WRKY26/38/43 & 15 & 16 & 19 & 15 & 15 & 25 & 24 & $4.164 e-56$ & 19 & 26 \\
\hline WRKY6 & 5 & 5 & 5 & 5 & 5 & 22 & 4 & $1.091 \mathrm{e}-10$ & 3 & 12 \\
\hline ZAP1 & 22 & 44 & 45 & 22 & 22 & 1 & 1 & $2.468 e-415$ & 219 & 268 \\
\hline
\end{tabular}

Promoter sequences from the top 600 induced and top 600 suppressed genes 10 dai were identified and their TFBS abundances quantified using Marina. A catalog of pre-assembled DNA motifs (1,160 motifs) and PWMs ( 80 matrices) accompanied such groups.

A total of 71 over-represented TFBSs were identified. Of these N TFBSs, magnitude of over-representation is ranked from 1 to $N$ such that the most over-represented are close to 1 while the least over-represented are close to $N$. Since TFBS models can vary across source-organisms, certain over-represented TFBSs were found multiple times (i.e. GAMYB, bZIP911, and ATHB5). Furthermore, not all metrics rank the same. As a result, manually deducing degree of TFBS over-representation can be a challenging task. IPF-standardization is designed to circumvent such a scenario.

Two gene-sets were then declared to contain the top 600 induced and 600 suppressed differentially expressed genes (DEGs), respectively. Per gene set, the promoter sequence $2.5 \mathrm{~kb}$ upstream from each genes transcription start site (TSS) was retrieved and appended to a FASTA file. Both FASTA files in-conjunction with 80 plant PWMs and 1,160 plant-specific DNA motifs served as input into Marina.

Marina identified 71 potentially over-represented TFBSs between the control and 10 dai groups (Table 4). As shown in this table, there exists no consensus amongst the various metrics as to which TFBS is truly the most over-represented. There are however some TFBSs that are ranked by all metrics in a relatively uniform manner:
AG, ATHB6, and ABFS. For all other TFBSs, it is difficult to deduce magnitude of over-representation. Such a scenario warrants IPF-standardization as it normalizes metric-ranks to agree in-concert which TFBSs are the most over-represented (Table 5). By visually contrasting this table with that of Table 4, it is clear that unstandardized ranks from Laplace Correction (LP), Confidence (CF) and Lift (LI) perfectly equal their IPF-standardized counterpart.

\section{Many over-represented TFBSs have defense or stress-response functions}

Given the list of IPF-standardized TFBSs (Table 5), all 4 WRKY genes were over-represented at 10 dai. These 
Table 5 IPF-standardized abundances provides agreement amongst all metrics

\begin{tabular}{|c|c|c|c|c|c|c|c|}
\hline \multirow[b]{2}{*}{ TF } & \multicolumn{7}{|c|}{ Metrics } \\
\hline & LP & $\mathrm{CO}$ & JAC & LI & CF & $\mathrm{K}$ & PHI \\
\hline ABF1 & 20 & 20 & 20 & 20 & 20 & 20 & 20 \\
\hline ABFS & 9 & 9 & 9 & 9 & 9 & 9 & 9 \\
\hline ABI3/FUS3 & 67 & 67 & 67 & 67 & 67 & 67 & 67 \\
\hline$A B \mid 4(2)$ & 64 & 64 & 64 & 64 & 64 & 64 & 64 \\
\hline$A G$ & 14 & 14 & 14 & 14 & 14 & 14 & 14 \\
\hline AGP1 & 48 & 48 & 48 & 48 & 48 & 48 & 48 \\
\hline ALFIN1 & 34 & 34 & 34 & 34 & 34 & 34 & 34 \\
\hline ARF1 & 65 & 65 & 65 & 65 & 65 & 65 & 65 \\
\hline ARR10 & 39 & 39 & 39 & 39 & 39 & 39 & 39 \\
\hline ARR2 & 69 & 69 & 69 & 69 & 69 & 69 & 69 \\
\hline ATHB-5 & 43 & 43 & 43 & 43 & 43 & 43 & 43 \\
\hline ATHB1 & 40 & 40 & 40 & 40 & 40 & 40 & 40 \\
\hline ATHB5-1 & 63 & 63 & 63 & 63 & 63 & 63 & 63 \\
\hline ATHB5-2 & 37 & 37 & 37 & 37 & 37 & 37 & 37 \\
\hline ATHB6 & 27 & 27 & 27 & 27 & 27 & 27 & 27 \\
\hline ATHB9 & 53 & 53 & 53 & 53 & 53 & 53 & 53 \\
\hline AtLEC2 & 56 & 56 & 56 & 56 & 56 & 56 & 56 \\
\hline ATML1/PDF2 & 71 & 71 & 71 & 71 & 71 & 71 & 71 \\
\hline AtMYB2 & 29 & 29 & 29 & 29 & 29 & 29 & 29 \\
\hline AtMYB77 & 60 & 60 & 60 & 60 & 60 & 60 & 60 \\
\hline AtMYC2 & 2 & 2 & 2 & 2 & 2 & 2 & 2 \\
\hline AtSPL3 & 30 & 30 & 30 & 30 & 30 & 30 & 30 \\
\hline BLR/RPL/PNY & 35 & 35 & 35 & 35 & 35 & 35 & 35 \\
\hline bZIP910(2) & 10 & 10 & 10 & 10 & 10 & 10 & 10 \\
\hline bZIP911 & 12 & 12 & 12 & 12 & 12 & 12 & 12 \\
\hline bZIP911(1) & 11 & 11 & 11 & 11 & 11 & 11 & 11 \\
\hline bZIP911(2) & 17 & 17 & 17 & 17 & 17 & 17 & 17 \\
\hline $\mathrm{CBF}$ & 43 & 43 & 43 & 43 & 43 & 43 & 43 \\
\hline CDC5 & 4 & 4 & 4 & 4 & 4 & 4 & 4 \\
\hline DOF2 & 42 & 42 & 42 & 42 & 42 & 42 & 42 \\
\hline DPBF1/2 & 51 & 51 & 51 & 51 & 51 & 51 & 51 \\
\hline $\mathrm{E} 2 \mathrm{Fa}$ & 70 & 70 & 70 & 70 & 70 & 70 & 70 \\
\hline $\mathrm{E} 2 \mathrm{Fc} / \mathrm{d}$ & 1 & 1 & 1 & 1 & 1 & 1 & 1 \\
\hline EmBP-1 & 25 & 25 & 25 & 25 & 25 & 25 & 25 \\
\hline GAMYB & 47 & 47 & 47 & 47 & 47 & 47 & 47 \\
\hline Gamyb & 58 & 58 & 58 & 58 & 58 & 58 & 58 \\
\hline GATA-1 & 18 & 18 & 18 & 18 & 18 & 18 & 18 \\
\hline GATA-1/2/3/4 & 16 & 16 & 16 & 16 & 16 & 16 & 16 \\
\hline GT-3b & 13 & 13 & 13 & 13 & 13 & 13 & 13 \\
\hline HAHB4 & 46 & 46 & 46 & 46 & 46 & 46 & 46 \\
\hline HAT5 & 43 & 43 & 43 & 43 & 43 & 43 & 43 \\
\hline HSE & 19 & 19 & 19 & 19 & 19 & 19 & 19 \\
\hline
\end{tabular}

Table 5 IPF-standardized abundances provides agreement amongst all metrics (Continued)

\begin{tabular}{|c|c|c|c|c|c|c|c|}
\hline $\mathrm{HVH} 21$ & 41 & 41 & 41 & 41 & 41 & 41 & 41 \\
\hline HY5 & 6 & 6 & 6 & 6 & 6 & 6 & 6 \\
\hline ID1 & 28 & 28 & 28 & 28 & 28 & 28 & 28 \\
\hline MYB.PH3(1) & 55 & 55 & 55 & 55 & 55 & 55 & 55 \\
\hline MYB.PH3(2) & 52 & 52 & 52 & 52 & 52 & 52 & 52 \\
\hline MYB98 & 62 & 62 & 62 & 62 & 62 & 62 & 62 \\
\hline $\mathrm{O} 2$ & 33 & 33 & 33 & 33 & 33 & 33 & 33 \\
\hline OsbHLH66 & 26 & 26 & 26 & 26 & 26 & 26 & 26 \\
\hline OsCBT & 3 & 3 & 3 & 3 & 3 & 3 & 3 \\
\hline P & 57 & 57 & 57 & 57 & 57 & 57 & 57 \\
\hline PCF2 & 61 & 61 & 61 & 61 & 61 & 61 & 61 \\
\hline PCF5 & 59 & 59 & 59 & 59 & 59 & 59 & 59 \\
\hline PEND & 31 & 31 & 31 & 31 & 31 & 31 & 31 \\
\hline PIF3(2) & 21 & 21 & 21 & 21 & 21 & 21 & 21 \\
\hline RAP2.2 & 66 & 66 & 66 & 66 & 66 & 66 & 66 \\
\hline RAV1(1) & 49 & 49 & 49 & 49 & 49 & 49 & 49 \\
\hline RAV1(2) & 38 & 38 & 38 & 38 & 38 & 38 & 38 \\
\hline STF1 & 24 & 24 & 24 & 24 & 24 & 24 & 24 \\
\hline TAC1 & 68 & 68 & 68 & 68 & 68 & 68 & 68 \\
\hline TaMYB80 & 54 & 54 & 54 & 54 & 54 & 54 & 54 \\
\hline TBP & 36 & 36 & 36 & 36 & 36 & 36 & 36 \\
\hline TEIL & 50 & 50 & 50 & 50 & 50 & 50 & 50 \\
\hline TGA1 & 23 & 23 & 23 & 23 & 23 & 23 & 23 \\
\hline TGA1a & 32 & 32 & 32 & 32 & 32 & 32 & 32 \\
\hline WRKY11 & 8 & 8 & 8 & 8 & 8 & 8 & 8 \\
\hline WRKY18/40/62 & 7 & 7 & 7 & 7 & 7 & 7 & 7 \\
\hline WRKY26/38/43 & 15 & 15 & 15 & 15 & 15 & 15 & 15 \\
\hline WRKY6 & 5 & 5 & 5 & 5 & 5 & 5 & 5 \\
\hline ZAP1 & 22 & 22 & 22 & 22 & 22 & 22 & 22 \\
\hline
\end{tabular}

By having all metrics agree as to magnitude of over-representation per TFBS, the investigator will have an easier time identifying TFBSs of interest. Much like unstandardized ranks (Table 4), standardized ranks also range from 1 to $N$ such that ranks in the vicinity of 1 are most over-represented while ranks in the vicinity of $N$ are least over-represented.

abundances are supported by numerous studies which show that WRKY genes are perceived upon PAMP signals or abiotic stressors [40-43]. WRKY genes drive defenseresponse by regulating NONEXPRESSOR OF PR1 (NPR1) expression by binding to W-box motifs in the NPR1 promoter. NPR1 protein binds with TGA TFs which regulate pathogenesis-response (PR) expression, hence providing a means of positively regulating SA-defense response [44-46].

Similar to WRKY, a bZIP family TFBS, HY5, was also over-represented 10 dai. Inextricably linked to photomorphogenesis, this TF is also known for its positive regulation of auxin signalling; a phytohormone which regulates 
defense response $[47,48]$. Through interactions with HY1 and MYC2, HY5 is able to regulate photomorphogenesis, ABA and JA signaling $[49,50]$.

Much like MYC2, AtMYB2 is not only over-represented at 10 dai but also plays a role in ABA-signaling. Microarray analyses on Arabidopsis plants with 35S:AtMYC2/ AtMYB2 over-expression constructs revealed induced expression of several ABA-regulated genes [51].

The GT (Trihelix) TF family member, GT-3b, was over-represented at 10 dai. Much is unknown about this TF family let alone GT-3b, however what is known is that many GT members, like HY5, regulate photomorphogenic signaling [52]. A recent study showed how GT-2a and GT-2b over-expression positively-regulates ABA-signaling [53]. Though an over-expressed GT-3b construct was not part of this recent study, translating findings from GT-2a and GT-2b over to GT-3b could reveal potentially novel insights into whether GT-3b plays a part in $\mathrm{ABA}$ and defense-signaling roles.

\section{Strong relationship between degree of TFBS over-representation and IPF-rank}

Due to the multi-dimensional nature of unstandardized TFBS ranks (Table 4), dimensionality reduction was performed to facilitate rank visualization on a $2 \mathrm{D}$ coordinate plane. To carry-out such analysis, Principle Component
Analysis (PCA) followed by bi-variate clustering was executed using the R library clusplot [54]. All 71 TFBSs were partitioned into 6 discrete clusters and labeled based on their respective IPF-standardized rank (Figure 2). Interestingly, there appears to be a strong relationship between the magnitude of TFBS over-representation and IPFstandardized rank. This suggests that IPF-standardization is suitable for deducing magnitude of over-represented TFBSs.

\section{Comparative software analysis}

Several actively-used software tools and web-interfaces are available to quantify TFBS over-representation $[14,15,18,55-57]$. We classified such tools into two classes: software that deduce TFBS over-representation given either 1) one promoter-sequence set or 2) at least two promoter-sequence sets. Marina falls into this latter class and as does a popular software tool, F-MATCH [13]. Both tools require two FASTA files as input such that one file serves as a query sequence-set while the other a baseline control. Degree of over-representation is therefore deduced by statistically contrasting TFBS overrepresentation across these two groups.

Both software tools were compared using three independent sets of promoter-sequences of varying sizes. Each of these three analyses encompassed

\section{Over-represented TFBS clusters}

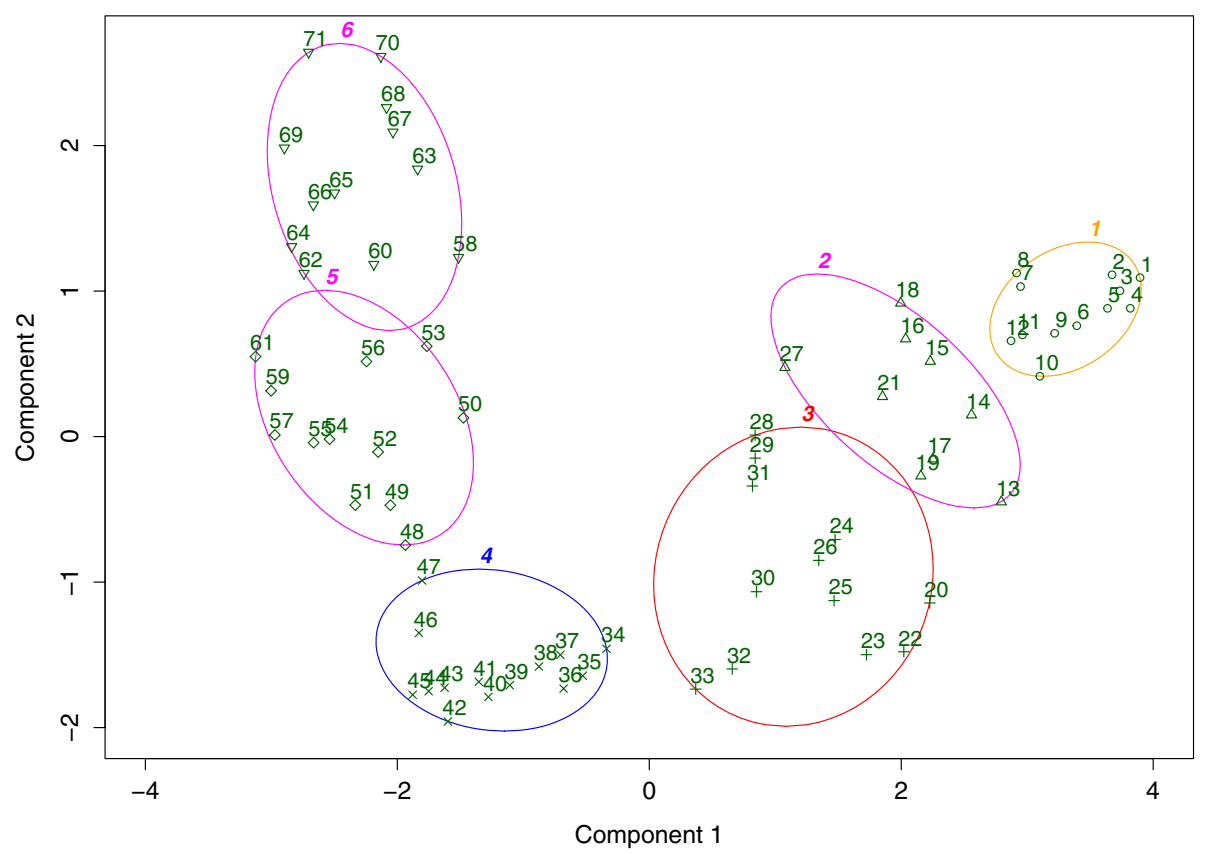

Figure 2 Clustering of over-represented TFBS. Performing dimensionality reduction on unstandardized TFBS ranks (Table 4) reveals distinct clusters of over-representative TFBSs. Each point in this 2-D coordinate plane references a unique TFBS, labeled based on its IPF-rank. From these 6 clusters, there appears to be a strong relationship between magnitude of TFBS over-representation and TFBS IPF-rank. The first two clusters, for instance, encapsulate all WRKY genes, GT-3b and HY5: genes perceived during defense response. This suggests that IPF-standardized ranks can elucidate magnitude of TFBS over-representation. 
Table 6 Comparing Marina and F-MATCH given catalogs of PWMs and DNA motifs

\begin{tabular}{ccccc}
\hline Group size & \multicolumn{2}{c}{ PWMs $(\mathbf{x} 80)$} & \multicolumn{2}{c}{ DNA motifs $(\times \mathbf{1 , 1 6 0})$} \\
(\# sequences) & F-MATCH & Marina & F-MATCH & Marina \\
\hline 600 & 44 & 47 & N/A & 24 \\
1500 & 0 & 50 & N/A & 41 \\
2500 & 0 & 53 & N/A & 44 \\
\hline
\end{tabular}

A collection of 80 plant-specific PWMs were supplied to Marina. When group-sizes are relatively small and PWMs are used, both Marina and F-MATCH identify approximately the same number of over-represented TFBSs. However as group-sizes increased, Marina consistently identifies over-represented TFBSs. Marina also accepts DNA motifs if PWMs are not available; F-MATCH does not accept such models.

promoter-sequences of DEGs 10 dai from our prior soybean - soybean rust RNA-Seq study [36]. F-MATCH and Marina identify relatively the same number of overrepresented TFBSs when promoter-sequence sets are 600 sequences in size (Table 6). As these promoter sets increase in size, Marina maintains steady consistency as to identification of over-representated TFBSs, while F-MATCH failed to detect any over-represented TFBSs. We believe the reasoning behind why F-MATCH yields 0 over-represented TFBSs while Marina identified almost 50 TFBSs to be attributed towards usage of the binomial distribution by F-MATCH, which is known to be sensitive to large test sets. As far as output consistency between the two tools, our only comparison pertains to results obtained with 600 sequences sets. Given the 44 F-MATCH and 47 Marina over-represented TFBSs, 21 TFBSs were shared between the two result-sets. Unlike F-MATCH, we did not include TRANSFAC Professional PWMs in our analysis. We believe by introducing such PWMs, the number of shared TFBSs would certainly increase.

\section{Conclusions}

Marina is a operating-system independent software tool to identify over-represented TFBSs across different groups of promoter sequences. We illustrate its usage using an RNA-Seq plant-pathogen study, however promoter sequences from any organism can be analyzed using Marina as long as compatible TFBS models are provided. We also show its capability to identify overrepresented TFBSs regardless of input size. Given large sets of DEGs, our results show that by contrasting their promoter sequences, TFBSs perceived during defense and stress response were significantly over-represented. Other lesser-known TFBSs joined these ranks, raising questions as to potential candidate TFs affiliated with defense-response.

The underlying algorithms within this tool are guided by a catalog of user-provided TFBS models be-it DNA motifs or PWMs. Thankfully, many regulatory element resources and databases exist. By contrasting this software tool to a popular alternative, we show that Marina is built for large promoter-sequence sets while being able to identify biologically sound over-representative TFBSs.

\section{Availability and requirements}

Project name: Marina.

Project home page: http://mason.gmu.edu/ phossein/ marina/

Operating system(s): Operating-system independent.

Programming language: Java version $7+$.

Other requirements: None.

License: BSD.

\section{Abbreviations}

ABA: Abscisic acid; CO: Cosine metric; CF: Confidence metric; DEG: Differentially expressed gene; IPF: Iterative proportional fitting; JA: Jasmonic acid; JAC: Jaccard; K: Cohen's kappa; LP: Laplace correction; LI: Lift; NPR1: Non-expressor of PR1; PHI: Phi-coefficient; PWM: Position weight matrix; SR: Soybean rust; TF: Transcription factor; TFBS: Transcription factor binding site.

\section{Competing interests}

The authors declare that they have no competing interests.

\section{Authors' contributions}

BFM inspired development of Marina. PH wrote the manuscript, implemented the Marina software tool and underlying algorithms. IO guided the development of PWM extrapolation and over-representation analyses. Al authors read, critiqued and approved the final manuscript.

\section{Acknowledgements}

We wish to thank the United States Department of Agriculture - Soybean Genomics and Improvement Laboratory (USDA - SGIL) for research funding and support. We also wish to thank Margaret MacDonald, Eric Brewer, Sara Kabir and Leila Taher for their comments on Marina functionality. Our appreciations also go out to Bret Cooper for advice on RNA-Seq quality analysis and correction. This research was supported in part by the Intramural Research Program of the National Institutes of Health, National Library of Medicine.

\section{Author details}

${ }^{1}$ Department of Bioinformatics and Computational Biology, George Mason University, Manassas, Virginia, USA. ${ }^{2}$ Computational Biology Branch, Nationa Center for Biotechnology Information, National Institutes of Health, Bethesda, Maryland, USA. ${ }^{3}$ Soybean Genomics and Improvement Laboratory, United States Department of Agriculture, Beltsville, Maryland, USA.

Received: 21 December 2012 Accepted: 28 March 2013 Published: 11 April 2013

\section{References}

1. Riaño-Pachón DM, Ruzicic S, Dreyer I, Mueller-Roeber B: PInTFDB: an integrative plant transcription factor database. BMC Bioinformatics 2007, 8:

2. Singh K, Foley RC, Oñate-Sánchez L: Transcription factors in plant defense and stress responses. Curr Opin Plant Biol 2002, 5(5):430-436.

3. Dubos C, Stracke R, Grotewold E, Weisshaar B, Martin C, Lepiniec L: MYB transcription factors in Arabidopsis. Trends Plant Sci 2010 15(10):573-581.

4. Carrasco JL, Ancillo G, Mayda E, Vera P: A novel transcription factor involved in plant defense endowed with protein phosphatase activity. EMBO 2003, 22:3376-3384.

5. Bulyk ML: Computational prediction of transcription-factor binding site locations. Genome Bio/ 2003, 5:

6. Cartharius K, Frech K, Grote K, Klocke B, Haltmeier M, Klingenhoff A, Frisch $M$, Bayerlein $M$, Werner T: MatInspector and beyond: promoter analysis based on transcription factor binding sites. Bioinformatics 2005, 21(13):2933-2942. 
7. Pertea M, Mount SM, Salzberg SL: A computational survey of candidate exonic splicing enhancer motifs in the model plant Arabidopsis thaliana. BMC Bioinformatics 2007, 8:159+

8. FauteuX F, Stromvik M: Seed storage protein gene promoters contain conserved DNA motifs in Brassicaceae, Fabaceae and Poaceae. BMC Plant Biol 2009, 9:126+.

9. Vandepoele $K$, Casneuf T, Van de Peer $Y$ : Identification of novel regulatory modules in dicotyledonous plants using expression data and comparative genomics. Genome Biol 2007, 7(11).

10. Leelavati N, Ovcharenko I: Identifying regulatory elements in eukaryotic genomes. Brief Funct Genomic Proteomic 2009, 8(4):215-230.

11. Chekmenev DS, Haid C, Kel AE: P-Match: transcription factor binding site search by combining patterns and weight matrices. Nucleic Acids Res 2005, 33:W432-W437.

12. Loots GG, Ovcharenko I, Pachter L, Dubchak I, Rubin EM: rVista for comparative sequence-based discovery of functional transcription factor binding sites. Genome Res 2002, 12(5):832-839.

13. Kel AE, Voss $N$, Jauregui $R$, Kel-Margoulis OV, Wingender E: Beyond microarrays: Finding key transcription factors controlling signal transduction pathways. BMC Bioinformatics 2006, 7(S-2)

14. Bülow L, Engelmann S, Schindler M, Hehl R: AthaMap, integrating transcriptional and post-transcriptional data. Nucleic Acids Res 2009, 37(Database-Issue):D983-D986.

15. Palaniswamy SK, James S, Sun H, Lamb RS, Davuluri RV, Grotewold E: AGRIS and AtRegNet: A platform to link cis-regulatory elements and transcription factors into regulatory networks. Plant Physio/ 2006, 140(3):818-829.

16. Rombauts S, Florquin $K$, Lescot $M, C$, Rouzé $P$, van de Peer, $Y$ : Computational approaches to identify promoters and cis-regulatory elements in plant genomes. Plant Physio/ 2003, 132(3):1162-1176.

17. Wingender E, Chen X, Fricke E, Geffers R, Hehl R, Liebich I, Krull M, Matys V, Michael H, Ohnhauser R, Pruss M, Schacherer F, Thiele S, Urbach S: The TRANSFAC system on gene expression regulation. Nucleic Acids Res 2001, 29:281-283.

18. Sandelin A, Alkema W, Engstrom P, Wasserman WW, Lenhard B: JASPAR: an open-access database for eukaryotic transcription factor binding profiles. Nucleic Acids Res 2007, 32:D91-D94

19. Gabdoulline RR, Eckweiler D, Kel AE, Stegmaier P: 3DTF: a web server for predicting transcription factor PWMs using 3D structure-based energy calculations. Nucleic Acids Res 2012,

40(Web-Server-Issue):180-185.

20. Horspool NR: Practical fast searching in strings. Softw Pract Exp 1980, 10(6):501-506

21. Ulmasov T, Hagen G, Guilfoyle TJ: ARF1, a transcription factor that binds to auxin response elements. Science 1997, 276: 1865-1868.

22. Ramakrishnan S, Rakesh A: Mining sequential patterns: generalizations and performance improvements. In Proceedings 5 th International Conference Extending Database Technology, EDBT. Avignon, France. London: Springer-Verlag; 1996:3-17.

23. Tan PN, Kumar $V$, Srivastava J: Selecting the right interestingness measure for association patterns. In Proceedings of the Eighth ACM SIGKDD International Conference on Knowledge Discovery and Data Mining, KDD '02. Edmonton, $A B$, Canada; 2002:32-41.

24. Geng L, Hamilton $\mathrm{HJ}$ : Interestingness measures for data mining: a survey. ACM Comput Surv 2006, 38(3).

25. Steinbach $M$, Tan PN, Xiong H, Kumar V: Objective measures for association pattern analysis. Contemp Math 2007, 443:205-226.

26. Agrawal R, Imielinski T, Swami A: Mining association rules between sets of items in large databases. In ACM SIGMOD Intl. Conf. Management of Data. Washington, D.C. New York: ACM; 1993:207-216.

27. Merceron A, Yacef $K$ : Interestingness measures for association rules in educational data. In Proceedings of Educational Data Mining 2008: 1st International Conference on Educational Data Mining. Montreal, Quebec; 2008:57-66

28. Jaccard P: Ètude comparative de la distribution florale dans une portion des Alpes et des Jura. Bull Société Vaudoise des Sci Naturelles 1901, 37:547-579.

29. Cohen J: A coefficient of agreement for nominal scales. Educ Psychol Meas 1960, 20:37-46.
30. Good IJ: booktitle=The Estimation of Probabilities: An Essay on Modern Bayesian Methods, Volume 30 of Research Monograph,. Cambridge: M.I.T. Press; 1965.

31. Brin S, Motwani R, Ullman JD, Tsur S: Dynamic itemset counting and implication rules for market basket data. In SIGMOD 1997, Proceedings ACM SIGMOD International Conference on Management of Data. Tucson, Arizona. New York: ACM; 1997:255-264

32. Cramér H: Mathematical Methods of Statistics. Princeton mathematical series: Princeton University Press; 1946.

33. Deming WE, Stephan FF: On a least squares adjustment of a sampled frequency table when the expected marginal totals are known. Ann Math Stat 1940, 11(4):427-444.

34. Mosteller F: Association and estimation in contingency tables. J Am Stat Assoc 1968, 63(321):1-28.

35. Tan PN, Kumar $\vee$, Srivastava J: Selecting the right objective measure for association analysis. Inf Syst 2004, 29:293-313.

36. Tremblay A, Hosseini P, Alkharouf NW, Li S, Matthews BF: Gene expression in leaves of susceptible Glycine max during infection with Phakopsora pachyrhizi using next generation sequencing. Sequencing 2011:14

37. Peterson JD, Umayam LA, Dickinson TM, Hickey EK, White O: The comprehensive microbial resource. Nucleic Acids Res 2001, 29: 123-125.

38. Li H, Durbin R: Fast and accurate short read alignment with Burrows-Wheeler transform. Bioinformatics 2009, 25(14):1754-1760.

39. Mortazavi A, Williams BA, McCue K, Schaeffer L, Wold B: Mapping and quantifying mammalian transcriptomes by RNA-Seq. Nat Methods 2008, 5(7):621-628.

40. Rushton PJ, Somssich IE, Ringler P, Shen QJ: WRKY transcription factors. Trends Plant Sci 2010, 15(5):247-258.

41. Eulgem T: Dissecting the WRKY web of plant defense regulators. PLoS Pathogy 2006, 2(11)

42. Eulgem T, Rushton PJ, Robatzek S, Somssich IE: The WRKY superfamily of plant transcription factors. Journal 2000, 5(5):199-206.

43. Eulgem T, Somssich IE: Networks of WRKY transcription factors in defense signaling. Curr Opin Plant Biol 2007, 10(4):366-371.

44. Pandey SP, Somssich IE: The role of WRKY transcription factors in plant immunity. Plant Physio/ 2009, 150(4):1648-1655.

45. Yu D, Chen C, Chen Z: Evidence for an important role of WRKY DNA binding proteins in the regulation of NPR1 gene expression. Plant Cell 2001, 13(7):1527-1540.

46. Dong X: NPR1, all things considered. Curr Opin Plant Biol 2004 7(5):547-552.

47. Sibout R, Sukumar P, Hettiarachchi C, Holm M, Muday GK, Hardtke CS: Opposite root growth phenotypes of hy 5 versus hy 5 hyh mutants correlate with increased constitutive auxin signaling. PLoS Genet 2004, 2(11).

48. Cluis CP, Mouchel CF, Hardtke CS: The Arabidopsis transcription factor HY5 integrates light and hormone signaling pathways. Plant 2004, 38(2):332-347.

49. Prasad BRVP, Kumar SV, Nandi A, Chattopadhyay S: Functional interconnections of HY1 with MYC2 and HY5 in Arabidopsis seedling development. BMC Plant Biol 2012, 12(37).

50. Boter M, Ruíz-Rivero O, Abdeen A, Prat S: Conserved MYC transcription factors play a key role in jasmonate signaling both in tomato and Arabidopsis. Journal 2004, 18(13):1577-1591.

51. Abe H, Urao T, Itom T, Seki M, Shinozaki K, Yamaguchi-Shinozaki K Arabidopsis AtMYC2 (bHLH) and AtMYB2 (MYB) function as transcriptional activators in abscisic acid signaling. Plant Cell 2003 15:63-78

52. Kaplan-Levy RN, Brewer PB, Quon T, Smyth DR: The trihelix family of transcription factors - light, stress and development. Trends Plant SCi 2012, 17(3):163-171.

53. Xie ZM, Zou HF, Lei G, Wei W, Zhou QY, Niu CF, Liao Y, Tian AG, Ma B, Zhang WK, Zhang JS, Chen SY: Soybean trihelix transcription factors GmGT-2A and GmGT-2B improve plant tolerance to abiotic stresses in transgenic Arabidopsis. PLOS ONE 2009, 4(9):e6898.

54. Pison G, Struyf A, Rousseeuw PJ: Displaying a clustering with CLUSPLOT. Comput Stat Data Anal 1999, 30(4):381-392.

55. Keilwagen J, Grau J, Paponov IA, Posch S, Strickert M, Grosse I: De-novo discovery of differentially abundant transcription factor binding 
sites including their positional preference. PLoS Comput Biology 2011, 7(2):e1001070+

56. Yamamoto YY, Obokata J: ppdb: a plant promoter database. Nucleic Acids Res 2008, 36(Database issue):D977-D981.

57. Chang WCC, Lee TYY, Huang HDD, Huang HYY, Pan RLL: PlantPAN: Plant promoter analysis navigator, for identifying combinatorial cis-regulatory elements with distance constraint in plant gene groups. BMC Genomics 2008, 9:561+.

doi:10.1186/1746-4811-9-12

Cite this article as: Hosseini et al.: Using an ensemble of statistical metrics to quantify large sets of plant transcription factor binding sites. Plant Methods 2013 9:12.

Submit your next manuscript to BioMed Central and take full advantage of:

- Convenient online submission

- Thorough peer review

- No space constraints or color figure charges

- Immediate publication on acceptance

- Inclusion in PubMed, CAS, Scopus and Google Scholar

- Research which is freely available for redistribution 